Review

International Journal of Medical Sciences

ISSN 1449-1907 www.medsci.org 2008 5(3):127-132

(C) Ivyspring International Publisher. All rights reserved

\title{
Adult neurogenesis, neuroinflammation and therapeutic potential of adult neural stem cells
}

\author{
Philippe Taupin 1,2
}

1. Fighting Blindness Vision Research Institute, National Institute for Cellular Biotechnology, Glasnevin. Dublin 9, Ireland.

2. Dublin City University, Dublin 9, Ireland.

Correspondence to: Philippe Taupin, Fighting Blindness Vision Research Institute. National Institute for Cellular Biotechnology. Dublin City University. Glasnevin. Dublin 9, Ireland. Email: philippe.taupin@dcu.ie

Received: 2008.04.12; Accepted: 2008.06.04; Published: 2008.06.05

The pathogenesis of neurological diseases and disorders remains mostly unknown. Neuroinflammation has been proposed as a causative factor for neurological diseases. The confirmation that neurogenesis occurs in the adult brain and neural stem cells (NSCs) reside in the adult central nervous system (CNS) of mammals has tremendous implications for our understanding of the physio- and pathology of the nervous system. The generation of newborn neuronal cells in the adult brain is modulated in neurological diseases and during inflammation. This suggests that adult neurogenesis is involved in the pathogenesis of neurological diseases and disorders, particularly during neuroinflammation. In this manuscript, we will review the modulation of adult neurogenesis in neurological diseases and during neuroinflammation. We will discuss the role and contribution of neuroinflammation and adult neurogenesis to neurological diseases and disorders, and for the therapeutic potential of adult NSCs.

Key words: neurogenesis, neuroinflammation, neural stem cells

\section{Introduction}

Neuroinflammation is a process in which the brain responds to infections, diseases and injuries [1, 2]. Neuroinflammation involve two types of immune cells: lymphocytes, monocytes and macrophages of the hematopoietic system, and microglial cells of the CNS $[3,4]$. Neuroinflammation disrupts the blood-brain barrier (BBB), allowing cells from the hematopoietic system to leave the blood stream and come in contact to the injury site [5]. The immune cells respond to injuries by eliminating debris and, synthesizing and releasing a host of powerful regulatory substances, like the complements, cytokines, chemokines, glutamate, interleukins, nitric oxide, reactive oxygen species and transforming growth factors [6-10]. The substances have both beneficial and harmful effects on the cellular environment, creating further damages [11] (fig. 1). Mature astrocytes are also activated following injury to the CNS $[12,13]$. Astrocytic activation is believed to be necessary for containing the immune response, repairing the $\mathrm{BBB}$ and attenuating further neuronal death $[5,14]$.

Contrary to a long-held dogma, neurogenesis occurs in the brain and NSCs reside in the CNS of adult mammals, in various species including human $[15,16]$. NSCs are the self-renewing multipotent cells that generate the main phenotypes of the nervous system. Neurogenesis is modulated in the brain of patients and in animal models of neurological diseases and disorders, like Alzheimer's disease (AD), epilepsy and Huntington's disease (HD) [17]. This suggests that the adult brain may be amenable to repair and that adult neurogenesis may contribute to the functioning, and phyio- and pathology of the CNS, particularly to the etiology of neurological diseases and disorders.

\section{Neuroinflammation in neurological diseases and injuries}

Inflammation is a process in which the body's white blood cells and chemicals protect us, from infections, foreign substances and injuries. In the CNS, neuroinflammation occurs following traumatic brain injuries, spinal cord injuries and cerebral strokes. It involves immune cells from the hematopoietic and nervous system $[1,2,6,18]$. It is now well documented that neuroinflammation is actively involved in neurological diseases and disorders, like $\mathrm{AD}$, amyotrophic lateral sclerosis, depression, epilepsy, HD, multiple sclerosis and Parkinson's disease (PD) [19-22]. Particularly, in AD, there is a correlation between local inflammation, and presence of amyloid plaques and neurofibrillary tangles [23].

It is proposed that chronic inflammation is a causative factor to the pathogenesis of neurological diseases and disorders [20, 24] (fig. 1). The immune 
cells and pro-inflammatory chemicals involved in neuroinflammation would underlie the mechanisms of diseases and neurodegeneration. The activation, or over activation, of immune cells involved in neuroinflammation and release of pro-inflammatory substances would result in reduced neuroprotection and neuronal repair, and increased neurodegeneration, leading to neurodegenerative diseases [10, 25, 26]. Depression is a common antecedent to many neurological diseases, particularly neurodegenerative diseases like $\mathrm{AD}$ and $\mathrm{PD}[27,28]$. Chronic inflammation during depressive episodes could predispose depressive patients to neurodegenerative diseases, later in life [29].

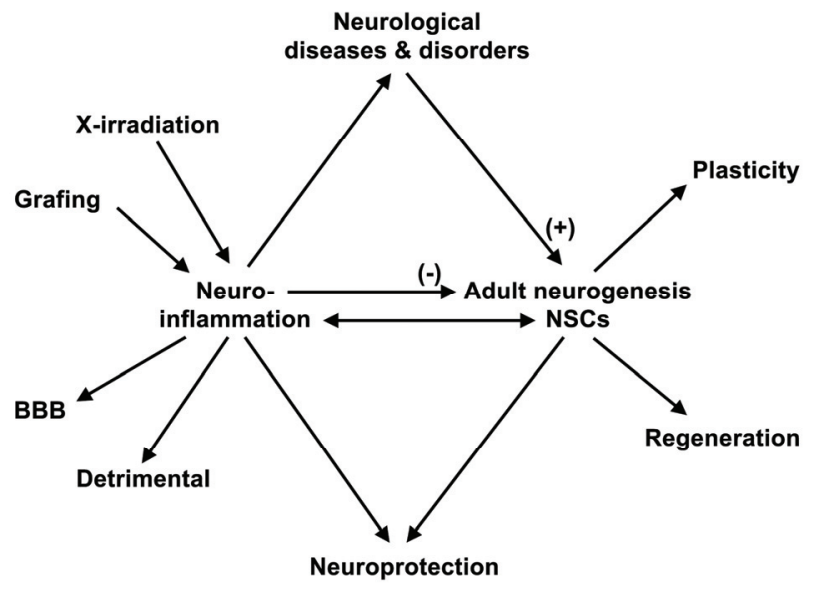

Figure 1. Adult neurogenesis and neuroinflammation. Neuroinflammation has been proposed as a causative factor for neurological diseases and disorders. It has both beneficial and harmful effects on the cellular environment. Neuroinflammation disrupts the BBB. Adult neurogenesis in modulated in a broad range of neurological diseases and disorders; it is decreased during inflammation. Adult neurogenesis may be involved in regenerative attempts and the plasticity of the nervous system. Adult-derived neural progenitor and stem cells grafted in the brain promote neuroprotection, by an immunomodulatory mechanism. Grafted neural progenitor and stem cells interact with the host immune system to promote functional recovery, an interaction that may provide clinical benefit for NSC-based therapy.

\section{Adult neurogenesis, neural stem cells and cellular therapy}

In the adult mammalian brain, including in humans, neurogenesis occurs primarily in two regions, the dentate gyrus (DG) of the hippocampus and subventricular zone (SVZ) [30, 31]. Neurogenesis involves a relatively small number of cells, particularly in the DG, and is modulated by environmental stimuli, trophic factors/cytokines, drug treatments, and in various physio- and pathological conditions, like neurological diseases and disorders [32]. Newborn neuronal cells, in the adult brain, establish functional connections, survive for extended period of time, at least 2 years in human, and reproduce processes similar to development, to integrate the mature network [30, 33, 34]. Adult neural progenitor and stem cells have been isolated and characterized in vitro, from various species [16], including from human biopsies and post-mortem tissues [35]. It is hypothesized that newborn neuronal cells in the adult brain originate from residual stem cells. The existence of stem cells in the adult brain suggests that it has the potential for self-repair and that newborn neuronal cells may contribute to the functioning, and physioand pathology of the CNS [36]. However, adult NSCs remain elusive cells and to be unequivocally identified and characterized in vitro and in vivo $[37,38]$.

Two strategies are being considered for adult NSC-based therapy in the CNS, the stimulation of endogenous neural progenitor or stem cells and the transplantation of adult-derived neural progenitor and stem cells [39]. Self-renewing multipotent neural progenitor and stem cells have been isolated and characterized in vitro, from various regions of the adult mammalian CNS, including the spinal cord [16]. This suggests that neural progenitor and stem cells reside throughout the adult $\mathrm{CNS}$, in mammals. The stimulation of endogenous neural progenitor or stem cells locally would represent a strategy to promote regeneration of the diseased and injured nervous system. Alternatively, new neuronal cells are generated at sites of degeneration in the diseased brain and after CNS injuries, like in HD and in experimental models of cerebral strokes 40,41]. These cells originate from the SVZ and migrate partially through the rostro-migratory stream to the sites of degeneration. This suggests that strategies to promote regeneration and repair may focus on stimulating SVZ neurogenesis. Adult derived-neural progenitor and stem cells may be transplanted locally [42] or administered intravenously to promote regeneration and repair [43]. Systemic injection provides a model of choice for delivering adult derived-neural progenitor and stem cells for the treatment of neurological diseases and injuries, where the degeneration is widespread, like AD and HD.

\section{Adult neurogenesis in neurological diseases and disorders}

Adult neurogenesis is modulated in the brains of patients and in animal models of neurological diseases and disorders, like AD, depression, epilepsy, Huntington's and Parkinson's diseases [17]. Neurogenesis is increased in the hippocampus of 
brains of patients with $\mathrm{AD}$, as revealed after autopsies by an increase in the expression of markers for immature neuronal cells, like doublecortin and polysialylated nerve cell adhesion molecule, in hippocampal regions [44]. In animal models of $\mathrm{AD}$, neurogenesis is increased in the DG of transgenic mice expressing the Swedish and Indiana amyloid protein precursor (APP) mutations, a mutant form of human APP, [45] and decreased in the DG and SVZ of knock-out mice for presenilin 1 and APP [46, 47]. This shows that adult neurogenesis is enhanced in $\mathrm{AD}$ brains. The discrepancies observed on adult neurogenesis in brain autopsies of patients with AD and animal models of $\mathrm{AD}$ may originate from the limitations of animal models, particularly transgenic mice, as representative models of complex diseases, particularly AD [48] and to study adult phenotypes, like adult neurogenesis. Result from autopsies reveals that neurogenesis is not altered in the brains of depressive patients [49]. Neurogenesis is enhanced in the DG and SVZ of animal models of epilepsy, like after pilocarpine treatment [50]. After pilocarpine treatment, ectopic granule-like cells in the hilus are labeled for bromodeoxyuridine (BrdU). BrdU is a thymidine analog that incorporates DNA of dividing cells during the S-phase of the cell cycle and is used for birthdating and monitoring cell proliferation [51]. MF-like processes immunostained for TOAD-64, a marker for newly generated neuronal cells, are also detected in the granule cell layer of the stratum oriens of CA3 and the inner molecular layer of the DG, in rodents [50]. Low-dose, whole-brain, $\mathrm{X}$-ray irradiation in adult rats, after pilocarpine treatment, inhibits neurogenesis, but does not prevent seizure-induced ectopic granule-like cells and MF sprouting [52]. Hence, neurogenesis is enhanced in the DG and SVZ in animal models of epilepsy and seizure-induced ectopic granule-like cells and MF sprouting arises not only from newborn neuronal cells, but also from mature dentate granule cells. Immunohistochemistry and confocal microscopy analysis of autopsies for markers of the cell cycle and neuronal differentiation, like proliferating cell nuclear antigen and $\beta$-tubulin, show that cell proliferation and neurogenesis are increased in the SVZ of brains of patients with HD [41]. In adult R6/1 transgenic mouse model of HD, neurogenesis decreases in the DG [53]. After quinolinic acid striatal lesioning of adult brain, neurogenesis is increased in the SVZ [54], as observed in brains of HD patients [41]. These data provide evidences that adult neurogenesis is increased in the SVZ of brains with HD. Data from R6/1 transgenic mouse model of $\mathrm{HD}$ are difficult to interpret in the context of adult neurogenesis in HD, as mutated forms of huntingtin affect brain development
[55]. This could underlie the decrease of neurogenesis reported in adult transgenic mice R6/1. In PD, one study reports that the rate of neurogenesis, measured by BrdU labeling, is stimulated in the substantia nigra (SN), following lesion induced by a systemic dose of MPTP (1-methyl-4-phenyl-1,2,3,6-tetrahydropyridine) [56]. Another study reports no evidence of new dopaminergic neurons in the $\mathrm{SN}$ of 6-hydroxydopamine-lesioned hemi-Parkinsonian rodents [57]. Hence, neurogenesis in the $\mathrm{SN}$ is the source of debates and controversies, and remains to be further evaluated.

In all, adult neurogenesis in modulated in a broad range of neurological diseases and disorders (fig. 1). The contribution and significance of this modulation to the etiology and pathogenesis of neurological diseases and disorders remain mostly unknown. In epilepsy, low-dose, whole-brain, X-ray irradiation in adult rats, after pilocarpine treatment, inhibits neurogenesis, but does not prevent the induction of recurrent seizures [52]. These data provide a strong argument against a critical role of adult neurogenesis in epileptogenesis. However, although increased hippocampal neurogenesis may not be critical to epileptogenesis, it could be a contributing factor to limbic seizures when present. In depression, chronic administration of antidepressants, like the selective serotonin reuptake inhibitors fluoxetine, increases neurogenesis in the DG, but not the SVZ in adult rats, suggesting that adult neurogenesis is involved in the activity of antidepressants [58, 59]. X-irradiation of the hippocampal region, but not other brain regions, like the SVZ or the cerebellar region, inhibits neurogenesis and prevents the behavioral effect of the antidepressants, like fluoxetine, in adult mice [60]. Hence, it is proposed that adult neurogenesis mediate the activities of antidepressants, particularly selective serotonin reuptake inhibitors. In HD, in brains of HD patients and after quinolinic acid striatal lesioning of adult brain the enhanced neurogenesis in the SVZ leads to the migration of neuroblasts and formation of new neuronal cells in damaged areas of the striatum. This suggests that neurogenesis may be involved in regenerative attempts in HD brains [41, 54] (fig. 1).

There are however debates and controversies over the modulation of adult neurogenesis in neurological diseases and disorders, particularly for studies involving BrdU labeling for studying neurogenesis. BrdU is a thymidine analog that incorporates DNA of dividing cells during the S-phase of the cell cycle and is used for birthdating and monitoring cell proliferation [51]. There are limitations and pitfalls over the use of BrdU for studying neurogenesis. BrdU is toxic and mutagenic substances. 
It triggers cell death, the formation of teratomes, alters DNA stability, lengthens the cell cycle, and has mitogenic, transcriptional and translational effects on cells that incorporate it. BrdU is not a marker for cell proliferation, but a marker for DNA synthesis [61-63]. High level, 4 to $10 \%$, of tetraploid nerve cells have been reported in regions in which degeneration occurs in $\mathrm{AD}$, like the hippocampus [64]. It is proposed that cell cycle re-entry and DNA duplication, without cell proliferation, precede neuronal death in degenerating regions of the CNS [65]. Some of the data observed by mean of immunohistochemistry for cell cycle proteins and BrdU labeling in the brains of AD patients and in animal models of the disease, may therefore not represent adult neurogenesis, but rather labeled nerve cells that may have entered the cell cycle and underwent DNA replication, but did not complete the cell cycle [62]. In addition, many physio- and pathological processes, like exercise, neurological diseases and injuries, like AD, PD and cerebral strokes and drugs treatments affect the permeability of the BBB and cerebral flow [66-68]. Some of the data observed by mean of BrdU labeling in animal models of neurological diseases and after drug treatments may reflect bio-availability of BrdU in the brain, rather than neurogenesis.

\section{Neuroinflammation in adult neurogenesis}

Neuroinflammation inhibits neurogenesis in the adult hippocampus [69, 70] (fig. 1). The mechanism, function and significance of the modulation of neurogenesis during inflammatory processes remain to be elucidated. Molecules released by the immune cells, like interleukins and nitric oxide, regulate negatively adult neurogenesis and may underlie the molecular mechanisms of inflammatory reactions on adult neurogenesis [71, 72]. Neuroinflammation is actively involved in neurological diseases and disorders, like AD, depression and PD [19-22]. It is proposed that chronic inflammation is a causative factor to the pathogenesis of these neurological diseases and disorders [20,24]. Hence, the modulation of adult neurogenesis during the inflammatory process may contribute or cooperate with the activity of neurological diseases and disorders on adult neurogenesis. Since the function of newborn neuronal cells is still the subject of debates and remains to be elucidated, the significance of the modulation of adult neurogenesis during inflammatory processes and in neurological diseases and disorders can only be speculated. Newborn neuronal cells may represent a regenerative attempt and contribute to the plasticity of the nervous system [73] (fig. 1).

There are however debates and controversies over the modulation of adult neurogenesis during inflammatory processes, particularly for studies involving BrdU labeling for studying neurogenesis. Neuroinflammation alters the permeability of the BBB [5]. Hence, some of the data observed by mean of BrdU labeling in animal models during inflammatory processes may reflect bio-availability of BrdU in the brain, rather than neurogenesis. Investigators have used X-ray irradiation to inhibit neurogenesis and study the function of adult neurogenesis [52, 60, 74]. Brain irradiation induces inflammatory responses (fig. 1). Hence, the effects of brain irradiation on adult neurogenesis in animal models, particularly of neurological diseases and disorders, are therefore difficult to interpret in light of these data. In all, the modulation of adult neurogenesis during inflammatory processes and after X-irradiation treatments remains to be further evaluated.

Neural progenitor and stem cells express receptors, and respond to trophic factors and cytokines. Hence, the inflammation resulting from the pathological processes to be treated by the transplantation of neural progenitor and stem cells, as well as the transplantation procedure itself may have adverse effects of the success of the graft (fig. 1). The timing of transplantation in the diseased brain or after injury is therefore critical for successful transplantation of neural progenitor and stem cell therapy [75]. Studies reveal that adult-derived neural progenitor and stem cells promote neuroprotection, by an immunomodulatory mechanism [76] (fig. 1). Grafted neural progenitor and stem cells interact with the host to promote functional recovery, an interaction that may provide clinical benefit for NSC-based therapy (fig. 1). The interaction of grafted neural progenitor and stem cells with the immune system suggests that pre-clinical studies involving immuno-depressed mice may not represent an appropriate model to characterize and validate sources of human-derived neural progenitor and stem cells for therapy [77].

\section{Conclusion and Perspectives}

Neuroinflammation is involved in the pathogenesis of neurological diseases and disorders, but its contribution and involvement to these pathological processes remain to be elucidated. It may be involved in the modulation of neurogenesis in neurological diseases and disorders, but the contribution and significance of this modulation remain to be understood. Neuroinflammation has tremendous implications for cellular therapy. On the one hand, it may limit the therapeutic potential of adult NSCs in vivo and ex vivo. On the other hand, it may interact with the neurogenic niches to promote the regenerative potential in vivo, and the integration of the grated neural progenitor and stem cells ex vivo. 
Hence, neuroinflammation may have both beneficial and detrimental effects on the potential of adult NSCs, to promote regeneration and repair in vivo and ex vivo. Therapeutic strategies for promoting the potential of adult NSCs in vivo and ex vivo may involve pro- and anti-inflammatory treatments. Future studies will aim at unraveling the molecular mechanisms governing the interaction between neural progenitor and stem cells and the immune system, and it implications for cellular therapy.

\section{Conflict of interest}

The author has declared that no conflict of interest exists.

\section{References}

[1] Nencini P, Sarti C, Innocenti R, et al. Acute inflammatory events and ischemic stroke subtypes. Cerebrovasc Dis. 2003; 15: 215-21.

[2] Schmidt OI, Heyde CE, Ertel W, Stahel PF. Closed head injury--an inflammatory disease? Brain Res. Brain Res Rev. 2005; 48: 388-99.

[3] Stoll G, Jander S. The role of microglia and macrophages in the pathophysiology of the CNS. Prog Neurobiol. 1999; 58: 233-47.

[4] Streit WJ, Walter SA, Pennell NA. Reactive microgliosis. Prog Neurobiol. 1999; 57: 563-81.

[5] Lossinsky AS, Shivers RR. Structural pathways for macromolecular and cellular transport across the blood-brain barrier during inflammatory conditions. Histol Histopathol. 2004; 19: 535-64.

[6] Ghirnikar RS, Lee YL, Eng LF. Inflammation in traumatic brain injury: role of cytokines and chemokines. Neurochem Res. 1998; 23: 329-40.

[7] Jander S, Schroeter M, Stoll G. Interleukin-18 expression after focal ischemia of the rat brain: association with the late-stage inflammatory response. J Cereb Blood Flow Metab. 2002; 22: 62-70.

[8] Stoll G, Schroeter M, Jander S, et al. Lesion-associated expression of transforming growth factor-beta- 2 in the rat nervous system: evidence for down-regulating the phagocytic activity of microglia and macrophages. Brain Pathol. 2004; 14: 51-8.

[9] Hensley K, Mhatre M, Mou S, et al. On the relation of oxidative stress to neuroinflammation: lessons learned from the G93A-SOD1 mouse model of amyotrophic lateral sclerosis. Antioxid Redox Signal. 2006; 8: 2075-87.

[10]Bonifati DM, Kishore U. Role of complement in neurodegeneration and neuroinflammation. Mol Immunol. 2007; 44: 999-1010.

[11]Stoll G, Jander S, Schroeter M. Detrimental and beneficial effects of injury-induced inflammation and cytokine expression in the nervous system. Adv Exp Med Biol. 2002; 513: 87-113.

[12] Latov N, Nilaver G, Zimmerman EA, et al. Fibrillary astrocytes proliferate in response to brain injury: a study combining immunoperoxidase technique for glial fibrillary acidic protein and radioautography of tritiated thymidine. Dev Biol. 1979; 72: 381-4.

[13] Miyake T, Hattori T, Fukuda M, et al. Quantitative studies on proliferative changes of reactive astrocytes in mouse cerebral cortex. Brain Res. 1988; 451: 133-8.

[14] Bush TG, Puvanachandra N, Horner $\mathrm{CH}$, et al. Leukocyte infiltration, neuronal degeneration, and neurite outgrowth after ablation of scar-forming, reactive astrocytes in adult transgenic mice. Neuron. 1999; 23: 297-308.

[15] Gross CG. Neurogenesis in the adult brain: death of a dogma. Nat Rev Neurosci. 2000; 1: 67-73.
[16] Taupin P, Gage FH. Adult neurogenesis and neural stem cells of the central nervous system in mammals. J Neurosci Res. 2002; 69:745-9.

[17] Grote HE, Hannan AJ. Regulators of adult neurogenesis in the healthy and diseased brain. Clin Exp Pharmacol Physiol. 2007; 34: 533-45.

[18] Stoll G, Jander S, Schroeter M. Inflammation and glial responses in ischemic brain lesions. Prog Neurobiol. 1998; 56: 149-71.

[19] Minghetti L. Role of inflammation in neurodegenerative diseases. Curr Opin Neurol. 2005; 18: 315-21.

[20] Eikelenboom P, Veerhuis R, Scheper W, et al. The significance of neuroinflammation in understanding Alzheimer's disease. J Neural Transm. 2006; 113: 1685-95.

[21] Hensley K, Mhatre M, Mou S, et al. On the relation of oxidative stress to neuroinflammation: lessons learned from the G93A-SOD1 mouse model of amyotrophic lateral sclerosis. Antioxid Redox Signal. 2006; 8: 2075-87.

[22] Klegeris A, Schulzer M, Harper DG, McGeer PL. Increase in core body temperature of Alzheimer's disease patients as a possible indicator of chronic neuroinflammation: a meta-analysis. Gerontology. 2007; 53: 7-11.

[23] Sivaprakasam K. Towards a unifying hypothesis of Alzheimer's disease: cholinergic system linked to plaques, tangles and neuroinflammation. Curr Med Chem. 2006; 13: 2179-88.

[24] Whitton PS. Inflammation as a causative factor in the aetiology of Parkinson's disease. Br J Pharmacol. 2007; 150: 963-76.

[25] Zilka N, Ferencik M, Hulin I. Neuroinflammation in Alzheimer's disease: protector or promoter? Bratisl Lek Listy. 2006; 107: 374-83.

[26] Donnelly DJ, Popovich PG. Inflammation and its role in neuroprotection, axonal regeneration and functional recovery after spinal cord injury. Exp Neurol. 2007; 209: 378-88.

[27] Karceski S. Early Parkinson disease and depression. Neurology. 2007; 69: E2-3.

[28] Potter GG, Steffens DC. Contribution of depression to cognitive impairment and dementia in older adults. Neurologist. 2007; 13: 105-17.

[29] Leonard BE. Inflammation, Depression and Dementia: Are they Connected? Neurochem Res. 2007; 32: 1749-56.

[30] Eriksson PS, Perfilieva E, Bjork-Eriksson T, et al. Neurogenesis in the adult human hippocampus. Nat Med. 1998; 4: 1313-7.

[31] Curtis MA, Kam M, Nannmark U, et al. Human neuroblasts migrate to the olfactory bulb via a lateral ventricular extension. Science. 2007; 315: 1243-9.

[32] Taupin P. Adult neurogenesis in the mammalian central nervous system: functionality and potential clinical interest. Med Sci Monit. 2005; 11: RA247-52.

[33] van Praag $\mathrm{H}$, Schinder AF, Christie BR, et al. Functional neurogenesis in the adult hippocampus. Nature. 2002; 415: 1030-4.

[34] Esposito MS, Piatti VC, Laplagne DA, et al. Neuronal differentiation in the adult hippocampus recapitulates embryonic development. J Neurosci. 2005; 25: 10074-86.

[35] Palmer TD, Schwartz PH, Taupin P, et al. Cell culture. Progenitor cells from human brain after death. Nature. 2001; 411:42-3.

[36] Taupin P. Adult neurogenesis in mammals. Curr Opin Mol Ther. 2006; 8: 345-51.

[37] Kornblum HI, Geschwind DH. Molecular markers in CNS stem cell research: hitting a moving target. Nat Rev Neurosci. 2001; 2: 843-6.

[38] Bull ND, Bartlett PF. 2005. The adult mouse hippocampal progenitor is neurogenic but not a stem cell. J Neurosci. 2005; 25: 10815-21.

[39] Taupin P. The therapeutic potential of adult neural stem cells. Curr Opin Mol Ther. 2006; 8: 225-31.

[40] Arvidsson A, Collin T, Kirik D, et al. Neuronal replacement 
from endogenous precursors in the adult brain after stroke. Nat Med. 2002; 8: 963-70.

[41] Curtis MA, Penney EB, Pearson AG, et al. Increased cell proliferation and neurogenesis in the adult human Huntington's disease brain. Proc Natl Acad Sci U S A. 2003; 100: 9023-7.

[42] Shihabuddin LS, Horner PJ, Ray J, Gage FH. 2000. Adult spinal cord stem cells generate neurons after transplantation in the adult dentate gyrus. J Neurosci. 2000; 20: 8727-35.

[43] Pluchino S, Quattrini A, Brambilla E, et al. Injection of adult neurospheres induces recovery in a chronic model of multiple sclerosis. Nature. 2003; 422: 688-94.

[44] Jin K, Peel AL, Mao XO, et al. Increased hippocampal neurogenesis in Alzheimer's disease. Proc Natl Acad Sci U S A. 2004; 101: 343-7.

[45] Jin K, Galvan V, Xie L, et al. Enhanced neurogenesis in Alzheimer's disease transgenic (PDGF-APPSw,Ind) mice. Proc Natl Acad Sci U S A. 2004; 101: 13363-7.

[46] Feng R, Rampon C, Tang YP, et al. Deficient neurogenesis in forebrain-specific presenilin-1 knockout mice is associated with reduced clearance of hippocampal memory traces. Neuron. 2001; 32, 911-26. Erratum in: Neuron. 2002; 33: 313.

[47] Wen PH, Shao X, Shao Z, et al. Overexpression of wild type but not an FAD mutant presenilin-1 promotes neurogenesis in the hippocampus of adult mice. Neurobiol Dis. 2002; 10: 8-19.

[48] Dodart JC, Mathis C, Bales KR, Paul SM. Does my mouse have Alzheimer's disease? Genes Brain Behav. 2002; 1: 142-55.

[49] Reif A, Fritzen S, Finger M, et al. Neural stem cell proliferation is decreased in schizophrenia, but not in depression. Mol Psychiatry. 2006; 11: 514-22.

[50] Parent JM, Yu TW, Leibowitz RT, et al. Dentate granule cell neurogenesis is increased by seizures and contributes to aberrant network reorganization in the adult rat hippocampus. J Neurosci. 1997; 17: 3727-38.

[51]Miller MW, Nowakowski RS. Use of bromodeoxyuridine-immunohistochemistry to examine the proliferation, migration and time of origin of cells in the central nervous system. Brain Res. 1988; 457: 44-52.

[52] Parent JM, Tada E, Fike JR, Lowenstein DH. Inhibition of dentate granule cell neurogenesis with brain irradiation does not prevent seizure-induced mossy fiber synaptic reorganization in the rat. J Neurosci. 1999; 19: 4508-19.

[53] Lazic SE, Grote H, Armstrong RJ, et al. Decreased hippocampal cell proliferation in R6/1 Huntington's mice. Neuroreport. 2004; 15: 811-3.

[54] Tattersfield AS, Croon RJ, Liu YW, et al. Neurogenesis in the striatum of the quinolinic acid lesion model of Huntington's disease. Neurosci. 2004; 127: 319-32.

[55] White JK, Auerbach W, Duyao MP, et al. Huntingtin is required for neurogenesis and is not impaired by the Huntington's disease CAG expansion. Nat Genet. 1997; 17: 404-10.

[56] Zhao M, Momma S, Delfani K, et al. Evidence for neurogenesis in the adult mammalian substantia nigra. Proc Natl Acad Sci U S A. $2003 ; 100: 7925-30$.

[57] Frielingsdorf H, Schwarz K, Brundin P, Mohapel P. No evidence for new dopaminergic neurons in the adult mammalian substantia nigra. Proc Natl Acad Sci U S A. 2004; 101: 10177-82.

[58] Malberg JE, Eisch AJ, Nestler EJ, Duman RS. Chronic antidepressant treatment increases neurogenesis in adult rat hippocampus. J Neurosci. 2000; 20: 9104-10.

[59] Malberg JE, Duman RS. Cell proliferation in adult hippocampus is decreased by inescapable stress: reversal by fluoxetine treatment. Neuropsychopharmacology. 2003; 28: 1562-71.

[60] Santarelli L, Saxe M, Gross C, et al. Requirement of hippocampal neurogenesis for the behavioral effects of antidepressants. Science. 2003; 301: 805-9.

[61] Nowakowski RS, Hayes NL. Stem cells: the promises and pitfalls. Neuropsychopharmacol. 2001; 25: 799-804.
[62] Taupin P. BrdU Immunohistochemistry for Studying Adult Neurogenesis: paradigms, pitfalls, limitations, and validation. Brain Res Rev. 2007; 53: 198-214.

[63] Taupin P. Protocols for Studying Adult Neurogenesis: Insights and Recent Developments. Regenerative Medicine. 2007; 2: 51-62.

[64] Yang Y, Geldmacher DS, Herrup K. DNA replication precedes neuronal cell death in Alzheimer's disease. J Neurosci. 2001; 21: 2661-8.

[65] Herrup K, Neve R, Ackerman SL, Copani A. Divide and die: cell cycle events as triggers of nerve cell death. J Neurosci. 2004; 24: 9232-9.

[66] Deane R, Zlokovic BV. Role of the blood-brain barrier in the pathogenesis of Alzheimer's disease. Curr Alzheimer Res. 2007; 4: 191-7.

[67] Desai BS, Monahan AJ, Carvey PM, Hendey B. Blood-brain barrier pathology in Alzheimer's and Parkinson's disease: implications for drug therapy. Cell Transplant. 2007; 16:285-99.

[68] Pereira AC, Huddleston DE, Brickman AM, et al. An in vivo correlate of exercise-induced neurogenesis in the adult dentate gyrus. Proc Natl Acad Sci U S A. 2007; 104: 5638-43.

[69] Ekdahl CT, Claasen JH, Bonde $S$, et al. Inflammation is detrimental for neurogenesis in adult brain. Proc Natl Acad Sci U S A. 2003; 100: 13632-7.

[70] Monje ML, Toda H, Palmer TD. Inflammatory blockade restores adult hippocampal neurogenesis. Science. 2003; 302: 1760-5.

[71] Vallieres L, Campbell IL, Gage FH, Sawchenko PE. Reduced hippocampal neurogenesis in adult transgenic mice with chronic astrocytic production of interleukin-6. J Neurosci. 2002; 22: 486-92 .

[72] Packer MA, Stasiv Y, Benraiss A, et al. Nitric oxide negatively regulates mammalian adult neurogenesis. Proc Natl Acad Sci U S A. 2003; 100: 9566-71.

[73] Taupin P. Adult neurogenesis and neuroplasticity. Restor Neurol Neurosci. 2006; 24: 9-15.

[74] Tada E, Parent JM, Lowenstein DH, Fike JR. X-irradiation causes a prolonged reduction in cell proliferation in the dentate gyrus of adult rats. Neurosci. 2000; 99: 33-41.

[75] Mueller FJ, McKercher SR, Imitola J, et al. At the interface of the immune system and the nervous system: how neuroinflammation modulates the fate of neural progenitors in vivo. Ernst Schering Res Found Workshop. 2005; 53: 83-114.

[76] Pluchino S, Zanotti L, Rossi B, et al. Neurosphere-derived multipotent precursors promote neuroprotection by an immunomodulatory mechanism. Nature. 2005; 436: 266-71.

[77] Taupin P. HuCNS-SC (StemCells). Curr Opin Mol Ther. 2006; 8: 156-63. 